\title{
Quasimonoenergetic laser plasma positron accelerator using particle-shower plasma-wave interactions
}

\author{
Aakash A. Sahai* \\ Department of Physics and John Adams Institute for Accelerator Science, Blackett Laboratory, \\ Imperial College London, SW7 2AZ, United Kingdom
}

(Received 15 January 2018; published 8 August 2018)

\begin{abstract}
An all-optical centimeter-scale laser-plasma positron accelerator is modeled to produce quasimonoenergetic beams with tunable ultrarelativistic energies. A new principle elucidated here describes the trapping of divergent positrons that are part of a laser-driven electromagnetic particle-shower with a large energy spread and their acceleration into a quasimonoenergetic positron beam in a laser-driven plasma wave. Proof of this principle using analysis and particle-in-cell simulations demonstrates that, under limits defined here, existing lasers can accelerate hundreds of $\mathrm{MeV}$ pC quasi-monoenergetic positron bunches. By providing an affordable alternative to kilometer-scale radio-frequency accelerators, this compact positron accelerator opens up new avenues of research.
\end{abstract}

DOI: 10.1103/PhysRevAccelBeams.21.081301

Monoenergetic positron accelerators intrinsic to positronelectron $\left(e^{+}-e^{-}\right)$colliders at energy frontiers $[1,2]$ have been fundamental to many important discoveries [3-6] that underpin the standard model. Apart from highenergy physics (HEP), monoenergetic $e^{+}$-beams of mostly sub-MeV energies are also used in many areas of material science [7,8], medicine [9] and applied antimatter physics [10]. Applications have however not had ready access to positron accelerators and have had to rely on alternative sources such as $\beta^{+}$-decay [11], $(\mathrm{p}, \mathrm{n})$ reaction [12] and pairproduction [13] of MeV-scale photons from-fission reactors [14], neutron-capture reactions [15] or MeV-scale $e^{-}$-beams impinging on a high-Z target [16].

Positron accelerators have evidently been scarce due to complexities involved in the production and isolation of elusive particles like positrons $[2,16]$ in addition to the costs associated with the large size of radio-frequency (rf) accelerators [17]. The size of conventional rf accelerators is dictated by the distance over which charged particles gain energy under the action of breakdown limited [18] tens of $\mathrm{MVm}^{-1} \mathrm{rf}$ fields sustained using metallic structures that reconfigure transverse electromagnetic waves into modes with axial fields. This limit also complicates efficient positron production $[2,13]$, which has required a multi-GeV $e^{-}$-beam from a kilometer-scale rf accelerator [17] to interact with a target. Furthermore, the positrons

\footnotetext{
Corresponding author. aakash.sahai@gmail.com

Published by the American Physical Society under the terms of the Creative Commons Attribution 4.0 International license. Further distribution of this work must maintain attribution to the author(s) and the published article's title, journal citation, and DOI.
}

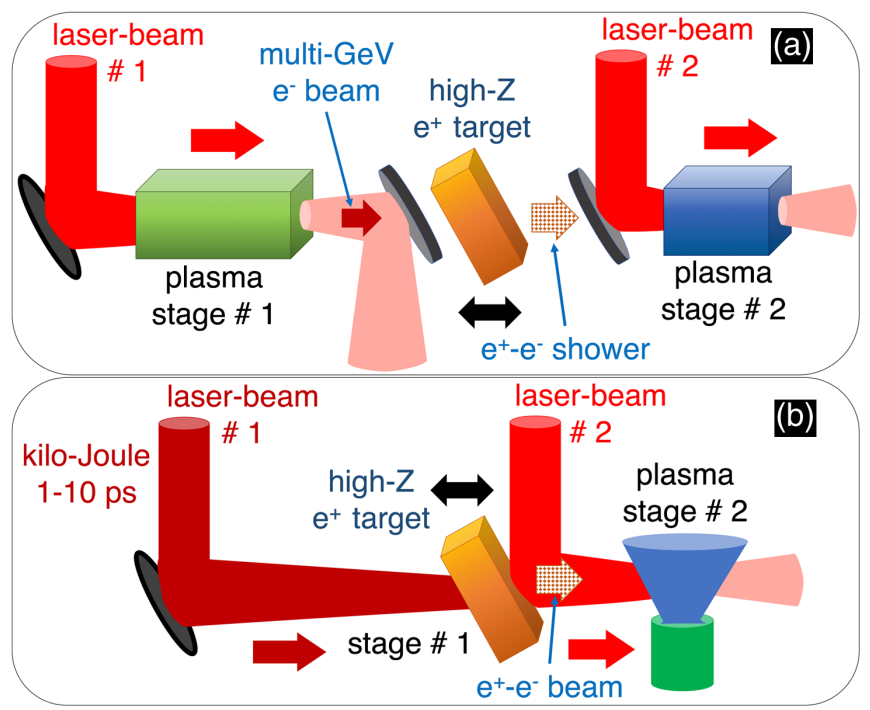

FIG. 1. Schematic of all-optical centimeter-scale schemes of quasimonoenergetic laser-plasma positron accelerator using the interaction of $e^{+}-e^{-}$showers with plasma-waves.

thus produced have to be captured in a flux concentrator, turned around and transported back [19] for reinjection into the same rf accelerator.

Advancements in $\mathrm{rf}$ technologies have demonstrated $100 \mathrm{MVm}^{-1}$-scale fields [20] but explorations beyond the standard model at TeV-scale $e^{+}-e^{-}$center-of-mass energies still remain unviable. Moreover, the progress of nonHEP applications of $e^{+}$-beams has been largely stagnant.

Recent efforts on compact and affordable positron accelerator design based on advanced acceleration techniques $[21,22]$ have unfortunately been unsatisfactory. Production of $e^{+}-e^{-}$showers using high-energy electrons from 


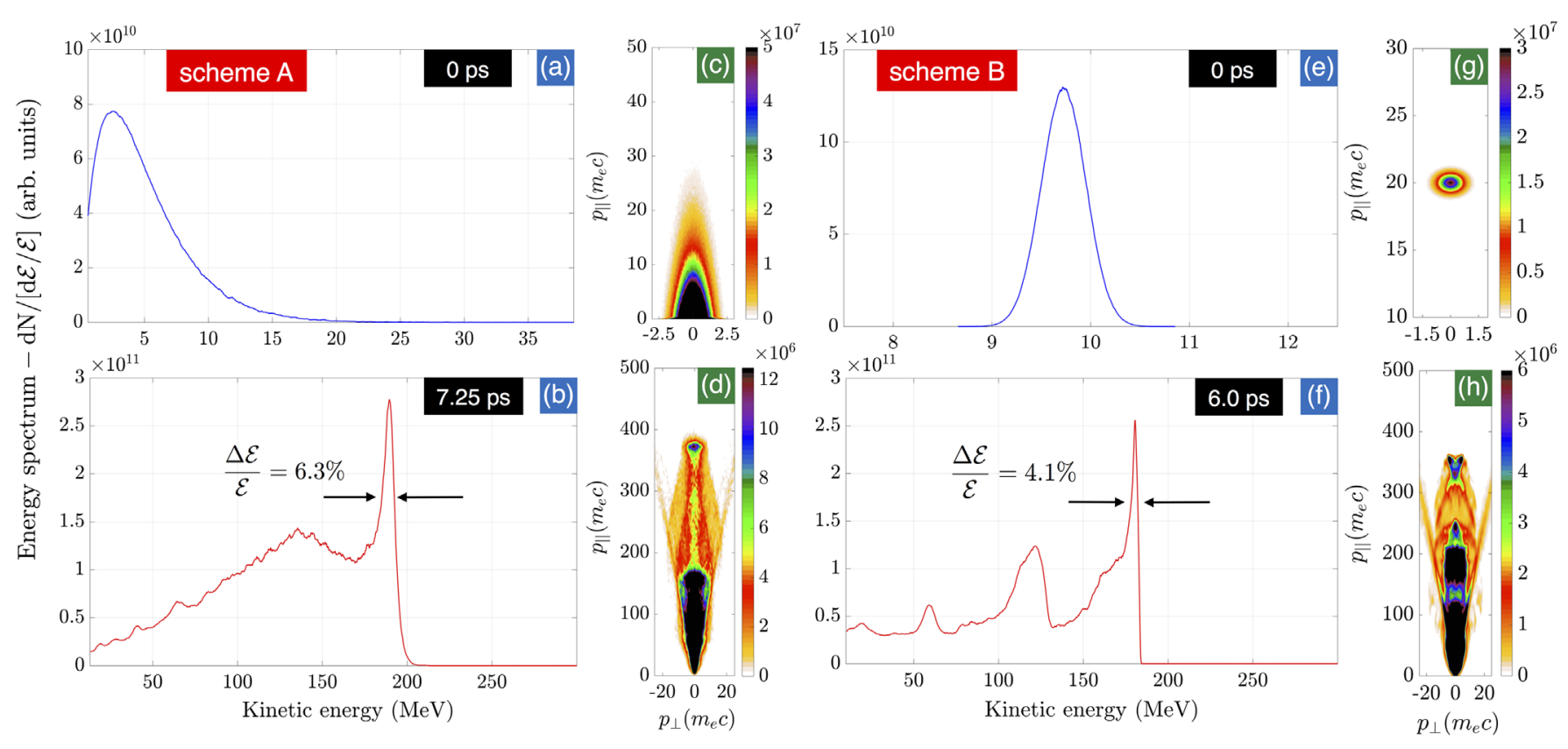

FIG. 2. Energy spectra and $p_{\perp}-p_{\|}$phase-spaces of $e^{+}$-LPA accelerated $e^{+}$-beams modeled with $n_{0}=10^{18} \mathrm{~cm}^{-3}$ using a $50 \mathrm{fs}$ laser with $a_{0}=1.4$ and Full Width at Half Maximum (FWHM) spot-size of $40 \mu \mathrm{m}$. For Scheme A (Scheme B), the initial conditions are in (a),(c) $[(\mathrm{e}),(\mathrm{g})]$ and the $e^{+}$-beam at $2.2 \mathrm{~mm}(1.8 \mathrm{~mm})$ in (b),(d) [(f),(h)].

compact laser-plasma accelerator $\left(e^{-}\right.$-LPA) [21-23] has been reported [24]. However, unlike $e^{+}$-"beams", showers are divergent and suffer from innately exponential energy spectra. Moreover, the positron number in showers which peaks around a few MeV [2,25], undergoes orders-ofmagnitude drop at higher energies. Another work which uses sheath fields driven by kilo-Joule (kJ) lasers in metal targets has obtained quasimonoenergetic $10 \mathrm{MeV}$ positrons [26] although with inherently high temperatures. Both scaling to higher energies and cooling of positrons using this mechanism is yet unexplored. Beam-driven plasma acceleration of positrons [27,28] although compact by itself, depends upon unviable kilometer-scale GeV rf accelerators. Additionally, obtaining an appropriately spaced drive-witness bunch pair for beam-plasma acceleration methods is technologically difficult.

In this paper, all-optical quasimonoenergetic $e^{+}$-beam production is proposed using a centimeter-scale positron accelerator (as shown in Fig. 1). This laser-plasma positron accelerator invention $\left(e^{+}\right.$-LPA) uses the interaction between laser-driven $e^{+}-e^{-}$particle showers [25] and laser-driven plasma waves that support $100 \sqrt{n_{0}\left(10^{18} \mathrm{~cm}^{-3}\right)} \mathrm{GVm}^{-1}$ fields $[21,22]\left(n_{0}\right.$ is the plasma electron density in $\left.\mathrm{cm}^{-3}\right)$. This letter models the trapping of divergent positrons that are part of laser-driven particle showers and their acceleration into a quasimonoenergetic $e^{+}$-beams, of tunable energy, in a laser-driven plasma wave.

This novel compact $e^{+}$-LPA opens up an affordable pathway for the application of ultrarelativistic quasimonoenergetic $e^{+}$-beams outside HEP as much as it invigorates research in advanced collider concepts [29].
The mechanism modeled in this paper uses two coupled laser-plasma interaction stages. In the first (positronproduction) stage, bremsstrahlung emission from laserdriven electrons undergoes pair-production in the nuclear Coulomb field inside a high-Z target and results in an electromagnetic-cascade particle-shower [13]. In scheme-A shown in Fig. 1(a), an $e^{-}$-LPA produces multi-GeVelectrons [30]. In scheme-B shown in Fig. 1(b), a kJ laser [26] produces an $\mathrm{MeV}$ electron flux in the pre-plasma of a solid target. The $e^{+}-e^{-}$shower from the target propagates into the second (positron-acceleration) stage where a significant number of shower particles are trapped in a laser-driven plasma-wave. The fields of the plasma-wave accelerate a quasimonoenergetic $e^{+}$-"beam" with typical energy spectra from particle-in-cell (PIC) simulations shown in Figs. 2(b) and 2(f). This groundbreaking quasimonoenergetic $e^{+}$-beam acceleration model defines the key principles as well as the limits of $e^{+}$-LPA. Recent efforts have shown that it is possible to overcome single-stage limits of electron acceleration using multistage $e^{-}$-LPAs albeit with a few technological challenges [31].

A proof of the principle of the above described $e^{+}$-LPA is developed below using analysis and PIC simulations.

The first stage laser-driven $e^{+}-e^{-}$showers are below modeled with characteristics that depend upon peak electron energy and net charge in scheme-A [25], laser energy in scheme-B [26] in addition to the target properties.

In scheme-A the particle-shower is modeled with an anisotropic relativistic Maxwellian distribution [32,33] consistent with experiments $[2,25]$. This distribution in momentum space (normalized to $\left.m_{e} c\right), \mathbf{p}=\left(p_{\perp}, p_{\|}\right)$is 


$$
f(\mathbf{p})=C\left(p_{\perp}^{2}+p_{\|}^{2}\right) \exp \left[-\beta_{\perp} \sqrt{1+p_{\perp}^{2}+A p_{\|}^{2}}\right]
$$

where $p_{\|}$is along the axis of laser propagation and $p_{\perp}$ in the transverse directions, $\beta_{\perp}=m_{e} c^{2} T_{\perp}^{-1}, A=T_{\|} T_{\perp}^{-1}$, transverse $T_{\perp}$ and longitudinal $T_{\|}$temperatures are in $\mathrm{eV}$ and $C$ normalizes the distribution [33]. Using experimental evidence $[2,25]$, the peak particle number is at $2.3 \mathrm{MeV}\left(d f(\mathbf{p}) / d p_{\|}=0\right)$ with $T_{\perp}=0.2 \mathrm{MeV}$ and $A=25$. The shower positron densities here lie between $10^{15}-10^{17} \mathrm{~cm}^{-3}$ with $e^{+}$-to- $e^{-}$density ratio $\left(f_{e^{+}} / f_{e^{-}}\right)$of between 0.1 to 0.4 [25].

Experiments on laser-driven $e^{+}-e^{-}$showers, which observed $10^{9}$ positrons over $1 \mathrm{MeV}$ [25] using $0.6 \mathrm{GeV}$ peak energy, $100 \mathrm{pC} e^{-}$-LPA electrons (with a $10 \mathrm{~J}, 50 \mathrm{fs}$, $\lambda_{0}=0.8 \mu \mathrm{m}$ wavelength laser) incident on 5-10 millimeter $\mathrm{Pb}$ target, showed excellent agreement with Monte-Carlo particle simulations (GEANT4/FLUKA). These simulations predict many times higher $e^{+}$-yield [34] using multi-GeV $e^{-}$-LPA electrons [30] but the innate distribution of showers in Eq. (1) is retained.

In scheme- $\mathrm{B}$, sheath-accelerated $e^{+}-e^{-}$shower is here modeled on experiments in [26] that observed $10^{10}$ positrons using a $305 \mathrm{~J}, \lambda_{0}=1.054 \mu \mathrm{m}, \tau_{p} \sim 10$ ps laser incident on millimeter-scale $A u$ targets. Here this quasimonoenergetic shower is modeled using a relativistically drifting Maxwellian distribution with a drift kinetic energy of $10 \mathrm{MeV}, T_{\perp}=T_{\|}=200 \mathrm{keV}$ (isotropically) and $10^{15}-10^{16} \mathrm{~cm}^{-3}$ densities with above $f_{e^{+}} / f_{e^{-}}$ratio.

Using the above shower models, trapping and acceleration of the shower positrons in a laser-driven plasma wave is analyzed below. The dependence of $e^{+}$-beam properties (energy spectrum, emittance, charge) on $e^{+}$-LPA secondstage parameters is also investigated.

In the electron compression phase of the wave, electronion charge-separation potential driven by the laser ponderomotive force [21,22] $\left(\propto \nabla\left(I_{0} \lambda_{0}^{2}\right)\right.$, where $I_{0}$ is the peak intensity of a laser) is found to trap, focus and accelerate the shower positrons. The difference in velocity of the shower positrons and the electron compression phase $\left(\beta_{\phi}=\right.$ $\left[1-\omega_{\mathrm{pe}}^{2} / \omega_{0}^{2}\right]^{\frac{1}{2}}, \omega_{0}=2 \pi c \lambda_{0}^{-1}, \omega_{\mathrm{pe}}=\left[4 \pi n_{0} e^{2} m_{e}^{-1}\right]^{\frac{1}{2}}$ is the electron plasma frequency) necessitates a careful analysis of their interaction. An analysis followed by PIC simulations below elucidates the requirements to trap shower positrons and tune the accelerated $e^{+}$-beam energy spectra and energy gain.

The threshold potential required to trap and retain a significant positron number can be analytically derived. The minimum kinetic energy, $\mathcal{E}_{\mathrm{sh}}=\left(\gamma_{\mathrm{sh}}-1\right) m_{e} c^{2}$ (labframe momentum, $\left.p_{\|}=\gamma_{\mathrm{sh}} \beta_{\mathrm{sh}}^{\|} m_{e} c, \gamma_{\mathrm{sh}}=\left[1-\beta_{\mathrm{sh}}^{2}\right]^{-\frac{1}{2}}\right)$ of the positrons that are trapped is chosen to be less than the peak of the distribution in Eq. (1). The Lorentz transformed lower-limit of trapped positron kinetic energy in wave-frame with $\gamma_{\phi}=\left[1-\beta_{\phi}^{2}\right]^{-\frac{1}{2}}=\omega_{0} / \omega_{\mathrm{pe}}$ is

$$
\mathcal{E}_{\mathrm{sh}}^{\prime}=\left(\frac{\omega_{0}}{\omega_{\mathrm{pe}}} \gamma_{\mathrm{sh}}\left(1-\beta_{\mathrm{sh}}^{\|} \beta_{\phi}\right)-1\right) m_{e} c^{2}
$$

Positrons with negative relative velocities in the wave frame at $\mathcal{E}_{\mathrm{sh}}^{\prime}$ are trapped only when a lower-limit of wave frame potential $\Psi^{\prime}$ is exceeded

$$
e \Psi^{\prime} \geq \mathcal{E}_{\mathrm{sh}}^{\prime}
$$

Lorentz transformation of the four potential $\left(\Psi^{\prime}, \mathbf{A}^{\prime}\right)\left(\mathbf{A}^{\prime}\right.$ is the wave vector potential) back to the lab-frame under gauge invariance gives the threshold potential $\Psi$ and $\psi_{\text {th }}$

$$
\begin{gathered}
\Psi=\frac{\omega_{\mathrm{pe}}}{\omega_{0}} \Psi^{\prime}+c \mathbf{A} \cdot \boldsymbol{\beta}_{\phi} \\
\psi_{\text {th }} \geq \gamma_{\mathrm{sh}}\left(1-\beta_{\mathrm{sh}}^{\|} \beta_{\phi}\right)-\frac{\omega_{\mathrm{pe}}}{\omega_{0}}, \psi=\frac{e \Psi}{m_{e} c^{2}}, A_{\|}=0 .
\end{gathered}
$$

The longitudinal trapping condition in Eq. (4) is necessary but not sufficient, because particles may still transversely escape. A threshold potential is therefore necessary to constrain the divergent positrons within a transverse escape momentum contour. This potential is derived by Lorentz transforming to the shower frame at $c \beta_{\mathrm{sh}}^{\|}$where the longitudinal momentum contracts and the average particle energy in the shower frame is $k_{B} T_{\perp}$. Thus, the threshold $\Psi^{\prime \prime}$ and $\psi_{\text {th }}$ are

$$
\begin{aligned}
e \Psi^{\prime \prime} & \geq \alpha k_{B} T_{\perp} \\
\psi_{\text {th }} & \geq \alpha \frac{k_{B} T_{\perp}\left(m_{e} c^{2}\right)^{-1}}{1+\mathcal{E}_{\mathrm{sh}}\left(m_{e} c^{2}\right)^{-1}}
\end{aligned}
$$

where, $\alpha>1$ accounts for the trapping of particles away from $c \beta_{\mathrm{sh}}^{\|}$in the shower momentum distribution.

The peak-shaped potential in the electron compression phase that satisfies Eqs. (4), (5) can be modeled as $\psi(\zeta, r)=-\psi_{0} \operatorname{sech}^{2}\left(\frac{\zeta-\zeta_{\text {peak }}}{L_{\Delta}}\right) \operatorname{sech}^{2}\left(\frac{r-r_{\text {peak }}}{R_{\Delta}}\right) \mathcal{H}(-\psi), \quad$ (using Eq. (20) in [22] and PIC data) where $\psi_{0}$ is the peak negative potential, $\mathcal{H}$ the step function and $\zeta=z-c \beta_{\phi} t$. This potential peaks at $\zeta_{\text {peak }}$ and $r_{\text {peak }}$ and falls off over scale-lengths, $L_{\Delta}\left(\psi_{0}\right)$ longitudinally and $R_{\Delta}\left(\psi_{0}\right)$ radially. The fields in this region are both accelerating and focussing.

The shape of the beam energy spectrum is optimized by restraining the potential (upper-limit), although Eq. (4) suggests its arbitrary increase to $\psi \gg 1$, can extend trapping to $p_{\mathrm{sh}}^{\|} \rightarrow 0$. Upon satisfaction of the trapping condition in Eqs. (4), (5), this work shows that it is the profile of the potential $\left[\psi_{0}, L_{\Delta}\left(\psi_{0}\right), R_{\Delta}\left(\psi_{0}\right)\right]$ which shapes the spectrum. This potential profile is dictated by the wave amplitude $\left(\delta n_{e} / n_{0}=n_{e} / n_{0}-1\right.$ where $n_{e}(\zeta, r)$ is the density in the wave) in accordance with $\nabla^{2} \psi=k_{\mathrm{pe}}^{2} \delta n_{e} / n_{0}$ [22] $\left(k_{\mathrm{pe}}=c^{-1} \omega_{\mathrm{pe}} / \beta_{\phi}\right)$. Dynamics studied here shows that as the wave steepens with increasing amplitude its positron 
acceleration phase shrinks, $L_{\Delta} \propto \psi_{0}^{-1}$. The resultant faster longitudinal field variation degrades the energy spectrum. As the positron trapping region size reduces, beam charge also decreases. A quasinonlinear wave with $\psi \sim \mathcal{O}(1)$ therefore turns out to be optimal.

Beam energy gain, $\Delta W$ is optimized as the distance of overlap between the trapped beam and the favorable potential maximizes. This acceleration length, $L_{\text {acc }}$ is shown to depend on the wave amplitude, $\psi_{0}$ and the plasma density, $n_{0}$. The wave amplitude is itself dictated by $n_{0}$ and the normalized laser vector potential, $a_{0}\left(=\max \left(\mathrm{e} \mathbf{A}_{0} / m_{e} c^{2}\right)\right)$ in accordance with $\delta n_{e} / n_{0} \propto$ $k_{\mathrm{pe}}^{-2} \nabla a_{0}^{2}$, while in plasma the $a_{0}$ is modified by the wave density as per $\left(\nabla^{2}-c^{-2} \partial^{2} / \partial t^{2}\right) a_{0}=k_{\mathrm{pe}}^{2}\left(n_{e} / n_{0}\right) a_{0}$ [22]. In this work, it is found that an initially high $a_{0}$ or a rise in $a_{0}$ due to laser evolution increases the wave amplitude which shortens the potential profile and constrains $L_{\text {acc }}$. This limit of the overlap dictates the energy gain $\Delta W=$ $e\left\langle E_{\|}\right\rangle_{L_{\text {acc }}} L_{\text {acc }}$ where $\left\langle E_{\|}\right\rangle_{L_{\text {acc }}}(=-\partial \psi / \partial \zeta)$ is the wave longitudinal field averaged over $L_{\text {acc }}$.

Multi-dimensional PIC simulations are used to validate the above analysis. Whereas 3D simulations (see Supplemental Material [35]) offer precision, parameter scans based on them demand inaccessible computational resources. Nevertheless, $2 \frac{1}{2} \mathrm{D}$ simulations adjusted to match $3 \mathrm{D}$ simulations allow for characterization over a wide parameter space. Here a 2D cartesian grid which resolves $\lambda_{0}=0.8 \mu \mathrm{m}$ with 25 cells in the longitudinal and 15 cells in the transverse direction tracks a linearly-polarized laser pulse at its group velocity. The above detailed particle shower model is initialized as shown in Figs. 2(a) and 2(c) [2(e) and 2(g)] for scheme-A (scheme-B). The shower transversely has $\sigma_{r}=25 \mu \mathrm{m}$ and longitudinally spans the entire box. Each particle species is initialized with 4 particles per cell. Absorbing boundary conditions are used for both fields and particles. The laser with a Gaussian envelope of length $50 \mathrm{fs}$ and $a_{0}(2 \mathrm{D})=2 a_{0}$ propagates in $50 \mu \mathrm{m}$ of free-space before it enters a fixed-ion plasma.

The results in Figs. 2 and 3 imply that the $e^{+}$-LPA modeled here accelerates quasimonoenergetic $e^{+}$-beams. The wave focusing fields segregate the $e^{+}$-beam from electrons (see Supplemental Material [35]). Over an $L_{\text {acc }} \simeq 2 \mathrm{~mm}, \Delta W \sim 200 \mathrm{MeV} e^{+}$-beams of around $5 \%$ FWHM energy spread $(\Delta \mathcal{E} / \mathcal{E})$ are accelerated using a quasi-nonlinear wave excited by a $6 \mathrm{~J}$ laser with $40 \mu \mathrm{m}$ FWHM spot-size in $n_{0}=10^{18} \mathrm{~cm}^{-3}$ as shown in Figs. 2(b) [2(f)] for scheme-A (scheme-B). These laser parameters chosen here in consideration of staging requirement of reflection off a plasma mirror [31]. Whereas using $\Delta W=$ $e\left\langle E_{\|}\right\rangle_{L_{\text {acc }}} L_{\text {acc }}$ the estimated $\left\langle E_{\|}\right\rangle_{L_{\text {acc }}} \simeq 100 \mathrm{GVm}^{-1}$ [22] is in excellent agreement with $100 \sqrt{n_{0}\left(10^{18} \mathrm{~cm}^{-3}\right)} \mathrm{GVm}^{-1}$, at $\gamma_{\phi} \simeq 42, L_{\text {acc }}$ is well below the expected dephasing length [22] and thus severely limits $\Delta W$. This limit on $L_{\text {acc }}$ is due to changes in laser properties during acceleration

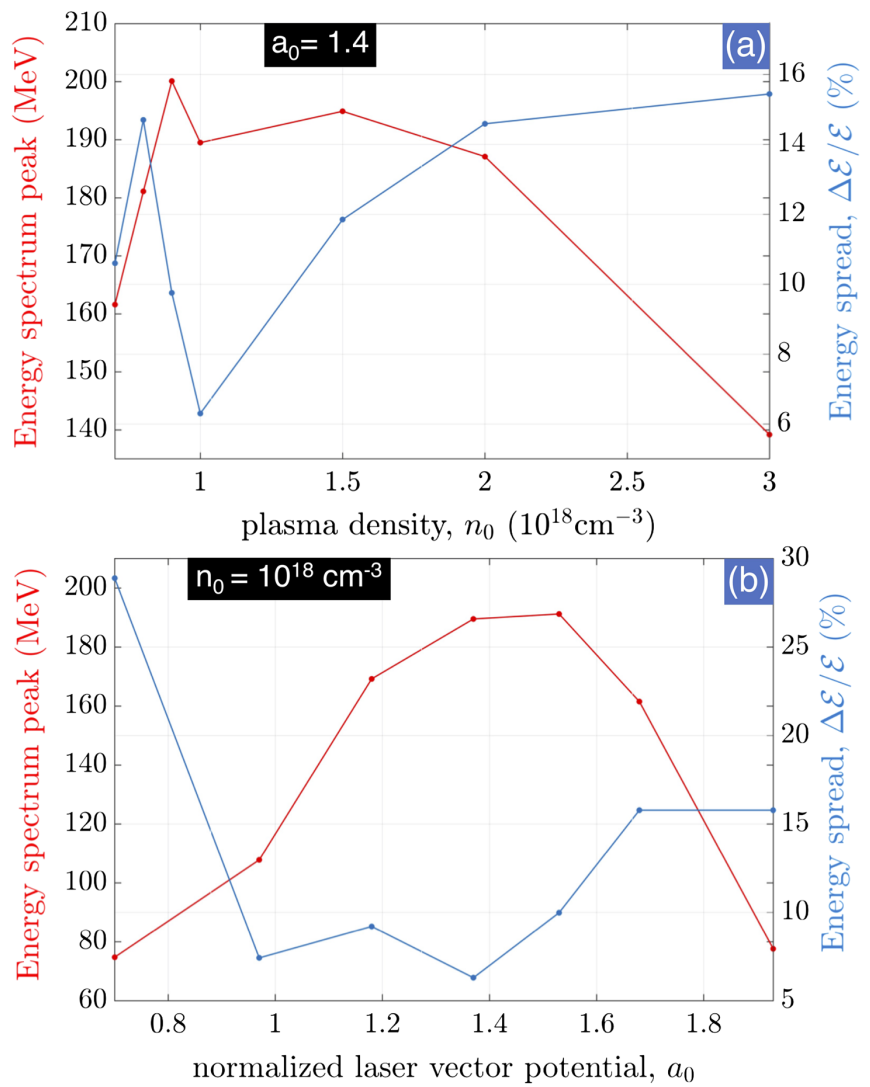

FIG. 3. Energy spectral characteristics of Scheme-A $e^{+}$-beam from PIC simulations varied with $n_{0}$ in (a) and $a_{0}$ in (b).

which modify the potential profile and the accelerating phase velocity and thrust the beam into defocusing ioncavity phase resulting in particle loss. This limit nonetheless motivates further work to better the energy gain, energy spread and bunch charge.

The $e^{+}$-beam confinement properties as inferred from the phase-space slices of scheme-A $e^{+}$-beam in Figs. 2(c) and 2(d) shown in Fig. 4 are remarkable. Bunch transverse size with $\sigma_{r}=5 \mu \mathrm{m}$ and length with $\sigma_{z}=7.5 \mu \mathrm{m}$ are estimated from Figs. 4(a), 4(c) and 4(b), 4(c), respectively. These bunch properties are consistent with Eq. (4) and (5). From Eq. (4) a threshold potential of $\psi_{\text {th }}=0.25$ at $n_{0}=$ $10^{18} \mathrm{~cm}^{-3}$ is required to trap positrons upto $\mathcal{E}_{\mathrm{sh}} \geq 0.5 \mathrm{MeV}$. This value of $\psi_{\text {th }}$ exceeds the Eq. (5) transverse threshold with $\alpha=2.5$. The observed bunch sizes are in excellent agreement with $L_{\Delta}$ and $R_{\Delta}$ of the simulated wave potential profiles. From $p_{\perp}-p_{\|}$slice in Fig. 2(c), the estimated opening angle of $\sim 15 \mathrm{mrad}$ can be further improved. From the real space in Fig. 4(c) a modest charge of $0.5-5 \mathrm{pC}$ is calculated.

The variation of $n_{0}$ at a fixed $a_{0}=1.4$ summarized in Fig. 3(a) implies that there is an optimal $n_{0}$ for a given intensity at which the peak beam energy maximizes and the energy spread minimizes. This optimality around $n_{0}=$ $10^{18} \mathrm{~cm}^{-3}$ is found to be due to the maximization of $L_{\text {acc }}$ 

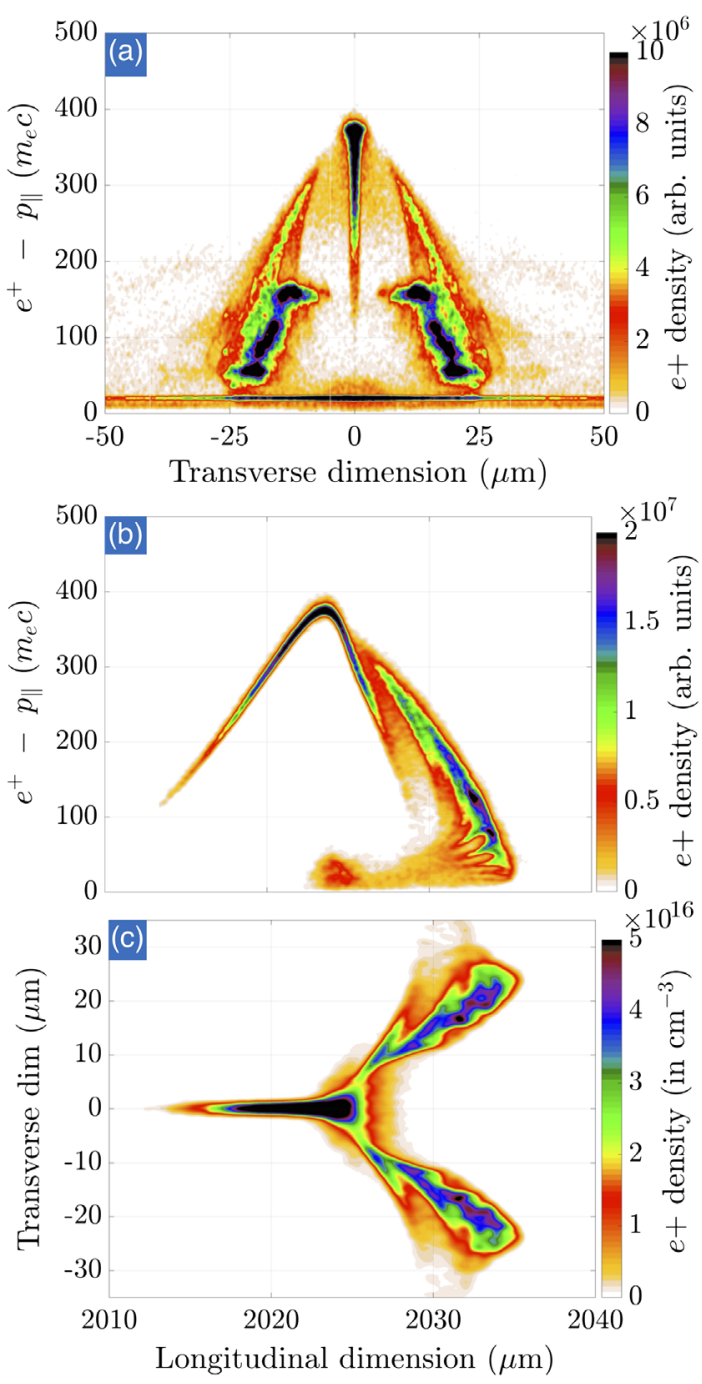

FIG. 4. Phase-space slices of scheme-A $e^{+}$-beam corresponding to Figs. 2(c) and 2(d)-(a) $p_{\|^{-}}$-transverse space, (b) $p_{\|^{-}}$ longitudinal space, (c) transverse-longitudinal space.

for the chosen laser parameters. At densities lower than the optimal smaller fields lead to slower energy gain, $\partial \Delta W / \partial z$ and weaker beam confinement while at higher densities the laser self-focuses too rapidly. In Fig. 3(b), $a_{0}$ is varied at fixed $n_{0}=10^{18} \mathrm{~cm}^{-3}$. An optimal quasinonlinear wave is found to be excited only around $a_{0}=1.4$ for the above shower and laser properties. At lower $a_{0}$ values the initially trapped positron number is small while for $a_{0}$ values higher than the optimal, steepened wave $\left(L_{\Delta} \rightarrow 0\right)$ accelerates beams with Maxwellian energy spectra.

In conclusion, this seminal work elucidates that control of particle-shower plasma-wave interaction, within certain limits identified here, enables all-optical acceleration of tunable hundreds of $\mathrm{MeV}$ quasimonoenergetic $e^{+}$-beams with $\mathrm{pC}$ charge using existing lasers. Future work will experimentally validate this $e^{+}$-LPA model, advance and explore novel high-energy antimatter applications and invent new schemes to overcome the key limits on $e^{+}$beam properties that have been identified in this invention (patent pending).

\section{ACKNOWLEDGMENTS}

This work was supported by the John Adams Institute for Accelerator Science and the Department of Physics at Imperial College London. The use of EPOCH PIC code developed in the U.K. is acknowledged. The simulations were initially performed using the Chanakya server at Duke University and subsequently using Imperial College High Performance Computing systems.

[1] B. Richter, Report No. SLAC-PUB-240, 1966; M. Tigner, A possible apparatus for electron clashing-beam experiments, Nuovo Cimento 37, 1228 (1965); J. R. Rees, Report No. SLAC-PUB-1911, 1977; Report No. SLAC-R-418a, 1993.

[2] R. Erikson, SLAC linear collider design handbook, Report No. SLAC-R-714, 1984, ch. 5, https://cds.cern.ch/record/ 105035/files/SLAC-R-714.pdf; J. R. J. Bennett, R. Billinge, M. H. Blewett, P. Brahmam et al., Design Concept for a $100 \mathrm{GeV} e^{+}-e^{-}$storage ring, Report No. CERN-7714, 1977, https://cds.cern.ch/record/118208/files/CERN77-14.pdf; LEP Collaboration, LEP design report, Report No. CERN-LEP-84-01, 1984.

[3] J.-E. Augustin, A. M. Boyarski, M. Breidenbach, F. Bulos, J. T. Dakin et al., Discovery of a Narrow Resonance in $e^{+} e^{-}$Annihilation, Phys. Rev. Lett. 33, 1406 (1974).

[4] M. L. Perl, G. S. Abrams, A. M. Boyarski, M. Breidenbach et al., Evidence for Anomalous Lepton Production in $e^{+}-e^{-}$Annihilation, Phys. Rev. Lett. 35, 1489 (1975).

[5] J. P. Lees et al. (BABAR Collaboration), Evidence for an Excess of $\bar{B} \rightarrow D^{(*)} \tau-\bar{\nu}_{\tau}$ Decays, Phys. Rev. Lett. 109, 101802 (2012).

[6] The ALEPH Collaboration, Precision electroweak measurements on the Z resonance, Phys. Rep. 427, 257 (2006); The LEP Collaboration, A combination of preliminary electroweak measurements and constraints on the standard model, arXiv:hep-ex/0412015; K. Abe et al., First measurement of the left-right cross section asymmetry in $\mathrm{Z}$ boson production by $e^{+} e^{-}$collisions, Phys. Rev. Lett. 70, 2515 (1993).

[7] A.P. Mills Jr., Surface analysis and atomic physics with slow positron beams, Science 218, 335 (1982); J. VanHouse and A. Rich, Surface investigations using the positron reemission microscope, Phys. Rev. Lett. 61, 488 (1988); A. David, G. Kogel, P. Sperr, and W. Triftshauser, Lifetime Measurements with a Scanning Positron Microscope, Phys. Rev. Lett. 87, 067402 (2001).

[8] M. Charlton and J.W. Humberston, Positron Physics, (Cambridge University Press, Cambridge, England, 2005), p. 454, ISBN: 9780521019392; M. Butterling, W. Anwand, T. E. Cowan, A. Hartmann, M. Jungmann, R. Krause-Rehberg, A. Krille, and A. Wagner, Gamma-induced Positron Spectroscopy (GiPS) at a super- 
conducting electron linear accelerator, Nucl. Instrum. Methods Phys. Res., Sect. B 269, 2623 (2011).

[9] D. E. Kuhl and R. Q. Edwards, Image Separation Radioisotope Scanning, Radiology 80, 653(1963); M. M. Ter-Pogossian, M. E. Phelps, E. J. Hoffman, and N. A. Mullani, A positron-emission transaxial tomograph for nuclear imaging (PETT), Radiology 114, 89 (1975).

[10] M. A. Kumakhov, On the theory of electromagnetic radiation of charged particles in a crystal, Phys. Lett. 57A, 17 (1976); W. Krause, A. V. Korol, A. V. Solov'yov, and W. Greiner, Spontaneous and stimulated undulator radiation by an ultra-relativistic positron channeling in a periodically bent crystal, Nucl. Instrum. Methods Phys. Res., Sect. A 475, 441 (2001); M. Amoretti et al., Production and detection of cold antihydrogen atoms, Nature (London) 419, 456 (2002); T. N. Wistisen, A. Di Piazza et al., arXiv:1704.01080.

[11] I. Curie and F. Joliot, Un nouveau type de radioactivite, Comptes Rendus Hebdomadaires de l'Academie des Sciences 198, 254 (1934); J. D. Cockroft, C. W. Gilbert, and E. T. S. Walton, , Nature (London) 133, 328 (1934).C. D. Ellis, and W. J. Henderson, Induced radioactivity of the lighter elements, Nature (London) 133, 530 (1934).

[12] J. M. Dawson, PPG-1022, Proc. of critical issues in the development of new linear colliders meeting, AIP Conf. Proc. 156, 194 (1987).

[13] H. Bethe and W. Heitler, On the stopping of fast particles and on the creation of positive electrons, Proc. R. Soc. A 146, 83 (1934); H. J. Bhabha and W. Heitler, The passage of fast electrons and the theory of cosmic showers, Proc. R. Soc. A 159, 432 (1937); L. Landau and G. Rumer, The cascade theory of electronic showers, Proc. R. Soc. A 166, 213 (1938).

[14] A. van Veen, H. Schut, and F. Labohm, and J. de Roode, Positron extraction and transport in a nuclear-reactor-based positron beam, Nucl. Instrum. Methods Phys. Res., Sect. A 427, 266 (1999); A. G. Hathaway, M. Skalsey, W. E. Frieze, R. S. Vallery, D. W. Gidley, A. I. Hawari, and $\mathrm{J}$. $\mathrm{Xu}$, Implementation of a prototype slow positron beam at the NC State University PULSTAR reactor, Nucl. Instrum. Methods Phys. Res., Sect. A 579, 538 (2007).

[15] B. Krusche and K. Schreckenbach, Intense positron sources by pair creation with neutron capture $\gamma$-rays, Nucl. Instrum. Methods Phys. Res., Sect. A 295, 155 (1990); C. Hugenschmidt, G. Koegel, R. Repper, K. Schreckenbach, P. Sperr, and W. Triftshauser, First platinum moderated positron beam based on neutron capture, Nucl. Instrum. Methods Phys. Res., Sect. B 198, 220 (2002).

[16] S. Ecklund, Report No. SLAC-PUB-4484, 1987; R. Krause-Rehberg, S. Sachert, G. Brauer, A. Rogov, and K. Noack, EPOS - An intense positron beam project at the ELBE radiation source in Rossendorf, Appl. Surf. Sci. 252, 3106 (2006); F. Bulos, H. DeStaebler, S. Ecklund, R. Helm, H. Hoag, H. Le Boutet, H. L. Lynch, R. Miller, and K. C. Moffeit, Design of a High Yield Position Source, IEEE Trans. Nucl. Sci. 32, 1832 (1985).

[17] R. Wideroe, Über ein neues Prinzip zur Herstellung hoher Spannungen, Archiv fur Elektrotechnik (Berlin) 21, 387 (1928); E. O. Lawrence and M. S. Livingston, The production of high speed light ions without the use of high voltages, Phys. Rev. 40, 19 (1932).

[18] W. D. Kilpatrick, Criterion for vacuum sparking designed to include both rf and dc, Rev. Sci. Instrum. 28, 824 (1957).

[19] G. Stange, A pulsed magnetic lens for positron focusing, numerical calculation and first measurements with a prototype, Internal Report No. DESY S 1-73/4, 1973, https://doi.org/10.1109/TNS.1979.4330728; A. V. Kulikov, S. D. Ecklund, and E. M. Reuter, SLC positron source pulsed flux concentrator, Proc. IEEE Particle Accelerator Conference ( IEEE, San Francisco, CA, USA, 1991), pp. 2005-2007, https://doi.org/10.1109/ PAC.1991.164851; R. H. Helm, J. E. Clendenin, S. D. Ecklund, A. V. Kulikov, and R. Pitthan, Isochronous 180 degrees turns for the SLC positron system, IEEE Particle Accelerator Conference (IEEE, San Francisco, CA, USA, 1991), pp. 500-502, https://doi.org/10.1109/ PAC.1991.164347; H. Braun, A. Kulikov, R. Pitthan, and M. Woodley, Nonlinear effects in the SLC $e^{+}$transport line, IEEE Particle Accelerator Conference, 1845 (IEEE, San Francisco, CA, USA, 1991), https://doi.org/10.1109/ PAC.1991.164797.

[20] C. Adolphsen, M. Barone, B. Barish, K. Buesser, P. Burrows et al., The International Linear Collider, Technical Design Report No. 3, https://arxiv.org/abs/1306.6328; P. Lebrun, L. Linssen, A. Lucaci-Timoce, D. Schulte, F. Simon, S. Stapnes et al., Compact Linear Collider, Conceptual Design Report (2012), Ch. 3, https://arxiv.org/pdf/ 1209.2543.pdf.

[21] T. Tajima and J. M. Dawson, Laser Electron Accelerator, Phys. Rev. Lett. 43, 267 (1979).

[22] E. Esarey, C. B. Schroeder, and W. P. Leemans, Physics of laser-driven plasma-based electron accelerators, Rev. Mod. Phys. 81, 1229 (2009); A. A. Sahai, Excitation of a nonlinear plasma ion wake by intense energy sources with applications to the crunch-in regime, Phys. Rev. Accel. Beams 20, 081004 (2017).

[23] S. P. D. Mangles et al., Monoenergetic beams of relativistic electrons from intense laser-plasma interactions, Nature (London) 431, 535 (2004); C. G. R. Geddes, Cs. Toth, J. van Tilborg, E. Esarey, C. B. Schroeder, D. Bruhwiler, C. Nieter, J. Cary, and W. P. Leemans, High-quality electron beams from a laser wakefield accelerator using plasma-channel guiding, Nature (London) 431, 538 (2004); J. Faure, Y. Glinec, A. Pukhov, S. Kiselev, S. Gordienko, E. Lefebvre, J.-P. Rousseau, F. Burgy, and V. Malka, A laser-plasma accelerator producing monoenergetic electron beams, Nature (London) 431, 541 (2004).

[24] G. Sarri, W. Schumaker, A. Di Piazza, M. Vargas et al., Table-Top Laser-Based Source of Femtosecond, Collimated, Ultrarelativistic Positron Beams, Phys. Rev. Lett. 110, 255002 (2013).

[25] G. Sarri, K. Poder, J. M. Cole, W. Schumaker et al., Generation of neutral and high-density electron-positron pair plasmas in the laboratory, Nat. Commun. 6, 6747 (2015).

[26] H. Chen, S. C. Wilks, D. D. Meyerhofer, J. Bonlie et al., Relativistic Quasimonoenergetic Positron Jets from Intense Laser-Solid Interactions, Phys. Rev. Lett. 105, 015003 (2010); H. Chen, S. C. Wilks, J. D. Bonlie, E. P. Liang, J. Myatt, D. F. Price, D. D. Meyerhofer, and P. Beiersdorfer, 
Relativistic Positron Creation Using Ultraintense Short Pulse Lasers, Phys. Rev. Lett. 102, 105001 (2009).

[27] S. Corde, E. Adli et al., Multi-gigaelectronvolt acceleration of positrons in a self-loaded plasma wakefield, Nature (London) 524, 442 (2015); B. E. Blue, C. E. Clayton, C. L. O'Connell, F.-J. Decker et al., Plasma-Wakefield Acceleration of an Intense Positron Beam, Phys. Rev. Lett. 90, 214801 (2003).

[28] X. Wang, R. Ischebeck, P. Muggli, T. Katsouleas, C. Joshi, W. B. Mori, and M. J. Hogan, Positron Injection and Acceleration on the Wake Driven by an Electron Beam in a Foil-and-Gas Plasma, Phys. Rev. Lett. 101, 124801 (2008); A. A. Sahai and T. C. Katsouleas, Optimal positron-beam excited plasma wakefields in Hollow and IonWake channels, in Proceedings of IPAC2015, Richmond, VA, USA (2015), http://accelconf.web.cern.ch/AccelConf/ IPAC2015/papers/wepje001.pdf.

[29] W. Leemans and E. Esarey, Laser-driven plasma-wave electron accelerators, Phys. Today 62, 44 (2009); A. Seryi et al., in Proceedings of the 23rd Particle Accelerator Conference, Vancouver, Canada, 2009 (IEEE, Piscataway, NJ, 2009), http://accelconf.web.cern.ch/AccelConf/ PAC2009/papers/WE6PFP081.pdf; C. B. Schroeder, E. Esarey, C. G. R. Geddes, C. Benedetti, and W. P. Leemans, Physics considerations for laser-plasma linear colliders, Phys. Rev. ST Accel. Beams 13, 101301 (2010).

[30] X. Wang, R. Zgadzaj, N. Fazel, Z. Li et al., Quasimonoenergetic laser-plasma acceleration of electrons to 2 GeV, Nat. Commun. 4, 1988 (2013); H. T. Kim, V. B. Pathak, K. H. Pae, A. Lifschitz et al., Stable multi-GeV electron accelerator driven by waveform-controlled PW laser pulses, Sci. Rep. 7, 10203 (2017).

[31] S. Steinke, J. van Tilborg, C. Benedetti, C. G. R. Geddes et al., Multistage coupling of independent laser-plasma accelerators, Nature (London) 530, 190 (2016); A. A. Sahai, Strongly mismatched regime of nonlinear laser plasma acceleration, arXiv:1711.00356.

[32] R. C. Davidson and P. H. Yoon, Nonlinear bound on unstable field energy in relativistic electron beams and plasmas , Phys. Fluids B 1, 195 (1989); P. H. Yoon, Relativistic Weibel instability, Phys. Plasmas 14, 024504 (2007); P. H. Yoon, Electromagnetic Weibel instability in a fully relativistic bi-Maxwellian plasma, Phys. Fluids B 1, 1336 (1989).

[33] R. A. Treumann and W. Baumjohann, Anisotropic Jüttner (relativistic Boltzmann) distribution, Ann. Geophys. 34, 737 (2016); S. Zenitani, Loading relativistic Maxwell distributions in particle simulations, Phys. Plasmas 22, 042116 (2015); O. Naito, A model distribution function for relativistic bi-Maxwellian with drift, Phys. Plasmas 20, 044501 (2013).

[34] G. Sarri and J. M. Cole (private communication).

[35] See Supplemental Material at http://link.aps.org/ supplemental/10.1103/PhysRevAccelBeams.21.081301 for the two movies presented in Supplementary Material show the evolution of (a) the real-space and (b) the momentum phase-space that underlie the controlled interaction of a positron-electron shower with laser-driven plasma-wave which leads to the trapping, focusing and acceleration of a quasi-monoenergetic positron beam and is the key physics in this invention. The movies demonstrate that this invention produces a positron-electron dual bunch configuration with micron-scale spatial separation but nearly same energy in the two bunches. Additional material in PDF format presents numerical and PIC-based solutions to the equations contained within the paper to further elucidate this invention. 